\title{
ON THE NUMBER OF CONJUGACY CLASS SIZES AND CHARACTER DEGREES IN FINITE $p$-GROUPS
}

\author{
GUSTAVO A. FERNÁNDEZ-ALCOBER AND ALEXANDER MORETÓ
}

\begin{abstract}
In this note we prove that for any two integers $r, s>1$ there exist finite $p$-groups $G$ of class 2 such that $|\operatorname{cd}(G)|=r$ and $|\operatorname{cs}(G)|=s$.
\end{abstract}

\section{INTRODUCTION}

In the last decades a number of results have been proved concerning the sets $\operatorname{cs}(G)$ of conjugacy class sizes and $\operatorname{cd}(G)$ of complex irreducible character degrees of a finite group $G$. Many of the results about class sizes are dual to results about character degrees, though the reason for the existence of this duality is not understood yet. For instance, Isaacs [?] proved that given any set $A$ of powers of a prime number $p$ containing 1 , there exists a $p$-group $G$ of class $\leq 2$ such that $\operatorname{cd}(G)=A$. The dual result for class sizes has been recently obtained in [?] by Cossey and Hawkes, who show that, for any set $A$ as above, there always exists a $p$-group of class $\leq 2$ such that $\operatorname{cs}(G)=A$. We raise more generally the following question: for which sets $A$ and $B$ of powers of $p$ containing 1 is it possible to find a $p$-group $G$ such that $\operatorname{cd}(G)=A$ and $\operatorname{cs}(G)=B$ ? Of course, if any of the two sets reduces to $\{1\}$ so does the other, hence we will assume that $|A|,|B| \geq 2$. Even in this case it is easy to see that there must be some kind of relation between $A$ and $B$. As already pointed out by Burnside [?, page 126], if $\operatorname{cs}(G)=\{1, p\}$ then $\left|G^{\prime}\right|=p$ and Theorem 7.5 in [?] yields that $\operatorname{cd}(G)=\left\{1,|G: Z(G)|^{1 / 2}\right\}$. On the other hand, according to Theorem 12.11 of [?], if $\operatorname{cd}(G)=\{1, p\}$ then either $G$ has an abelian maximal subgroup or $|G: Z(G)|=p^{3}$ and consequently $|\operatorname{cs}(G)| \leq 3$. In this note we prove that, surprisingly enough, there is not any relation between the number of character degrees and the number of class sizes.

Theorem. Given any two integers $r$ and s greater than 1 there exists a p-group $G$ of class 2 such that $|\operatorname{cd}(G)|=r$ and $|\operatorname{cs}(G)|=s$.

The proof of this theorem is elementary, but still we think that the result is noteworthy and, together with the examples above, indicates that it may be very difficult to find a complete answer to the question raised.

Most of the work of the second author was done while he was visiting the University of Wisconsin-Madison. He would like to thank the Mathematics Department for its hospitality and I.M. Isaacs for encouraging us to think about this question. The authors also want to express their thanks to A. Jaikin-Zapirain for helpful discussions.

1991 Mathematics Subject Classification. Primary 20C15, 20 D15.

Research of the second author supported by a grant of the Basque Government and by the University of the Basque Country grant UPV 127.310-EB160/98. 


\section{Proof of the TheOrem}

We first prove that, for any $n>1$, there exist $p$-groups of class 2 with $n$ character degrees and 2 class sizes and also groups with $n$ class sizes and 2 character degrees. The theorem will then be a straightforward consequence of these results.

Let us denote by $\mathfrak{D}_{p}$ the variety of $p$-groups of class $\leq 2$ and exponent $p$ when $p$ is odd, and the variety generated by the dihedral group of order 8 when $p=2$. In our next lemma we see that the free groups in this variety provide examples for our first case.

Lemma 2.1. Let $F_{n}$ be the free group of rank $n \geq 2$ in the variety $\mathfrak{D}_{p}$. Then $\operatorname{cd}\left(F_{n}\right)=\left\{1, p, \ldots, p^{[n / 2]}\right\}$ and $\operatorname{cs}\left(F_{n}\right)=\left\{1, p^{n-1}\right\}$.

Proof. As observed in [?], any two elements of $F_{n}$ that are independent modulo $\Phi\left(F_{n}\right)$ do not commute and, on the other hand, $\Phi\left(F_{n}\right)=Z\left(F_{n}\right)$ has index $p^{n}$ in $F_{n}$. We derive from these facts that $\operatorname{cs}\left(F_{n}\right)=\left\{1, p^{n-1}\right\}$.

Let us examine now the character degrees of $F_{n}$. Since $\left|F_{n}: Z\left(F_{n}\right)\right|=p^{n}$, we deduce from Corollary 2.30 of [?] that $\chi(1) \leq p^{[n / 2]}$ for any complex irreducible character $\chi$ of $F_{n}$. Conversely, choose any positive integer $i \leq[n / 2]$. Since $F_{2 i}$ is a quotient of $F_{n}$, it suffices to show that $p^{i} \in \operatorname{cd}\left(F_{2 i}\right)$. If we consider an appropriate maximal subgroup $N$ of $F_{2 i}^{\prime}$ then $F_{2 i} / N$ is an extraspecial group of order $p^{2 i+1}$ and, according to [?, Example 7.6], $p^{i} \in \operatorname{cd}\left(F_{2 i} / N\right) \subseteq \operatorname{cd}\left(F_{2 i}\right)$. The result follows.

For the second case, we need an explicit construction. Recall from [?, Lemma 5.7] that if $K$ and $L$ are finite groups such that $\operatorname{cd}(K)=\{1, m\}, \operatorname{cd}(L)=\{1, n\}$ and $K^{\prime} \cong L^{\prime}$, then any product $G$ of $K$ and $L$ with $K^{\prime}$ and $L^{\prime}$ amalgamated satisfies that $\operatorname{cd}(G)=\{1, m n\}$.

Lemma 2.2. Let $K_{n}=\langle b\rangle\left[\left\langle a_{1}\right\rangle \times \cdots \times\left\langle a_{n}\right\rangle\right]$ be a semidirect product, where $b$ has order $p$, each $a_{i}$ has order $p^{2}$ and the action of $b$ is given by $a_{i}^{b}=a_{i}^{1+p}$. If $G_{l, n}$ denotes the canonical central product of $l \leq n$ copies of $K_{n}$ then $\operatorname{cd}\left(G_{l, n}\right)=\left\{1, p^{l}\right\}$ and $\operatorname{cs}\left(G_{l, n}\right)=\left\{1, p, \ldots, p^{l}, p^{n}\right\}$.

Proof. The result about $\operatorname{cd}\left(G_{l, n}\right)$ follows from the remark before the lemma, since $\operatorname{cd}\left(K_{n}\right)=\{1, p\}$ by Ito's Theorem [?, Theorem 6.15]. We prove the claim about $\operatorname{cs}\left(G_{l, n}\right)$ by induction on $l$. For $l=1, G_{1, n}=K_{n}$ and the result is clear. We assume now that $l>1$ and $\operatorname{cs}\left(G_{l-1, n}\right)=\left\{1, p, \ldots, p^{l-1}, p^{n}\right\}$.

We have that $G_{l, n}$ is the quotient of the direct product $T=G_{l-1, n} \times K_{n}$ by the normal subgroup $N=\left\{\left(x, x^{-1}\right) \mid x \in Z\left(K_{n}\right)\right\}$, after identifying the centre of $G_{l-1, n}$ with the centre of $K_{n}$. Let us use the bar notation in $G_{l, n}$. Since the class of an element does not increase its size when passing to a quotient, for $x \in G_{l-1, n}$, $y \in K_{n}$ we have that

$$
\left|\mathrm{Cl}_{G_{l, n}}(\overline{(x, y)})\right| \leq\left|\mathrm{Cl}_{T}((x, y))\right|=\left|\mathrm{Cl}_{G_{l-1, n}}(x)\right|\left|\mathrm{Cl}_{K_{n}}(y)\right| .
$$

On the other hand,

$$
\begin{aligned}
\left|\mathrm{Cl}_{G_{l, n}}(\overline{(x, y)})\right| & \geq\left|\left\{\overline{(x, y)} \overline{\overline{(g, 1)}} \mid g \in G_{l-1, n}\right\}\right| \\
& =\left|\left\{\overline{\left(x^{g}, 1\right)} \mid g \in G_{l-1, n}\right\}\right|=\left|\mathrm{Cl}_{G_{l-1, n}}(x)\right|
\end{aligned}
$$

and similarly $\left|\mathrm{Cl}_{G_{l, n}}(\overline{(x, y)})\right| \geq\left|\mathrm{Cl}_{K_{n}}(y)\right|$. Therefore

$$
\max \left\{\left|\mathrm{Cl}_{G_{l-1, n}}(x)\right|,\left|\mathrm{Cl}_{K_{n}}(y)\right|\right\} \leq\left|\mathrm{Cl}_{G_{l, n}}(\overline{(x, y)})\right| \leq\left|\mathrm{Cl}_{G_{l-1, n}}(x)\right|\left|\mathrm{Cl}_{K_{n}}(y)\right| \text {. }
$$


If we use (??) with $y=1$, we deduce that the size of the class of $\overline{(x, 1)}$ in $G_{l, n}$ is the same as that of the class of $x$ in $G_{l-1, n}$. Hence $\left\{1, p, \ldots, p^{l-1}, p^{n}\right\} \subseteq \operatorname{cs}\left(G_{l, n}\right)$. On the other hand, if we take into acount that $\operatorname{cs}\left(K_{n}\right)=\left\{1, p, p^{n}\right\}$, the induction hypothesis and that the size of a class in $G_{l, n}$ is at most $\left|G_{l, n}^{\prime}\right|=p^{n}$, it also follows from (??) that $\operatorname{cs}\left(G_{l, n}\right) \subseteq\left\{1, p, \ldots, p^{l}, p^{n}\right\}$.

So the theorem will be proved once we show that $p^{l} \in \operatorname{cs}\left(G_{l, n}\right)$. Let $x$ be an element whose class in $G_{l-1, n}$ has size $p^{l-1}$. Since $Z\left(K_{n}\right)=K_{n}^{\prime}$ is an elementary abelian group of order $p^{n}$ generated by the commutators $\left[a_{i}, b\right]$ and $l-1<n$, some $\left[a_{i}, b\right]$ is not contained in $\left[x, G_{l-1, n}\right]$. Then

$$
\left|\mathrm{Cl}_{G_{l, n}}\left(\overline{\left(x, a_{i}\right)}\right)\right|=\left|\overline{\left[x, G_{l-1, n}\right] \times\left[a_{i}, K_{n}\right]}\right|=p^{l}
$$

as we wanted to prove.

Finally, we proceed to prove our theorem. In the proof, $E_{n}$ will stand for an extraspecial group of order $p^{2 n+1}$ and $U_{n}$ will denote a Sylow $p$-subgroup of $S L\left(3, p^{n}\right)$. Then $\left|U_{n}\right|=p^{3 n}$ and, according to [?, Lemma 4], $U_{n}$ is a semiextraspecial group (that is, the quotient by any maximal subgroup of the centre is extraspecial) with centre of order $p^{n}$. It follows from Theorem A in [?] that $\operatorname{cd}\left(U_{n}\right)=\operatorname{cs}\left(U_{n}\right)=\left\{1, p^{n}\right\}$.

Proof of the Theorem. If $r \leq s$ it suffices to consider the group $G=G_{n, n} \times E_{n} \times$ $\stackrel{r-2}{\cdots} \times E_{n}$, where $n=s-r+1$.

Assume now that $s \leq r$. If $s=2 l$ is even then for any $n \geq l$ the group $G=F_{2 n} \times E_{1} \times \stackrel{l-1}{\cdots} \times E_{1}$ satisfies that $|\operatorname{cs}(G)|=2 l$ and $|\operatorname{cd}(G)|=n+l$, so we are done by choosing $n=r-l$. If $s=2 l+1>3$ is odd then for $n \geq l$ the group $G_{l-1,2 n} \times F_{2 n+1}$ has $2 l+1$ class sizes and $n+l$ character degrees, and again it suffices to take $n=r-l$. Lastly, for $s=3$ consider the groups $F_{2 n+1} \times F_{2 n+1}$ and $U_{2 n} \times F_{2 n+1}$, where $n \geq 1$. The number of class sizes is 3 for any of these groups and, on the other hand, the number of character degrees is respectively $2 n+1$ and $2 n+2$, hence it may equal any $r \geq 3$.

\section{REFERENCES}

[1] B. Beisiegel, Semi-extraspezielle p-Gruppen, Math. Z. 156 (1977), 247-254.

[2] W. Burnside, "Theory of Groups of Finite Order", Dover, New York, 1955.

[3] J. Cossey, T. Hawkes, Sets of p-powers as conjugacy class sizes, Proc. Amer. Math. Soc. 128 (2000), 49-51.

[4] G.A. Fernández-Alcober, A. Moretó, Groups with two extreme character degrees and their normal subgroups, to appear in Trans. Amer. Math. Soc.

[5] B. Huppert, "Character Theory of Finite Groups", de Gruyter, Berlin/New York, 1998.

[6] I.M. IsaAcs, Sets of p-powers as irreducible character degrees, Proc. Amer. Math. Soc. 96 (1986), 551-552.

[7] I.M. IsAacs, "Character Theory of Finite Groups", New York, Dover, 1994.

[8] I.M. Isaacs, D.S. Passman, A characterization of groups in terms of the degrees of their characters II, Pacific J. Math 24 (1968), 467-510.

Departamento de Matemáticas, Universidad del País Vasco, 48080 Bilbao (Spain)

E-mail address: mtpfealg@lg.ehu.es

Departamento de Matemáticas, Universidad del País Vasco, 48080 Bilbao (Spain)

E-mail address: mtbmoqua@lg.ehu.es 